\title{
Acquisition of the Surface Morphology of Ruled Gratings with a Microscope Objective
}

\author{
Z.H. Li ${ }^{a, b}$, L. MA ${ }^{a}$, C.X. LiU ${ }^{a}$, M.N. ZhAnG ${ }^{c}$, X.Y. Chen ${ }^{d}$ And C.F. Cheng ${ }^{a, *}$ \\ ${ }^{a}$ College of Physics and Electronics, Shandong Normal University, Jinan 250014, China \\ ${ }^{b}$ College of Physics and Electric Information, Dezhou University, Dezhou 253023, China \\ ${ }^{c}$ College of Science, Qilu University Of Technology, Jinan 250353, China \\ ${ }^{d}$ School of Physics and Technology, University of Jinan, Jinan 250022, China
}

(Received February 27, 2014; in final form March 22, 2015)

\begin{abstract}
In this paper, an optical microscope objective with large numerical number is inserted into a Mach-Zehnder interferometer, and this system is adopted to detect the surface morphologies of two ruled transmission gratings with area scale to a micrometer. The object waves transmitting from the gratings interfere with spherical reference wave, and the interferograms constructed are recorded by a high-resolution CCD. The surface maps of the gratings are retrieved from the interferograms, and the results are confirmed by the measurement with an atomic force microscope, with detection errors in nanometer scale. This work provides an optical non-destructive method for precise detection of small-area sophisticated object surfaces with an optical microscope objective.
\end{abstract}

DOI: 10.12693/APhysPolA.127.1630

PACS: 42.30.Kq, 42.87.-d, 42.79.Dj, 42.40.Kw

\section{Introduction}

In the past few decades, the developments in many applied fields, e.g., semiconductor devices [1, 2], surface plasmas $[3,4]$, fractals [5] and surface materials, have been closely dependent on surface morphology, and researchers have proposed many methods to detect and describe the surface morphologies [6]. The most commonly used ones in nowadays are the atom force microscope and the scanning tunneling microscope [7], and high resolution is realized therein. However, such devices are expensive, the detection process may damage the object surfaces, and the scope of application is limited. Another way to detect surface morphology is using optical methods, e.g., the equal thickness interference [8] and the X-ray technique [9], where the morphology information is carried by the transmitting or reflected light that is not destructive. In practice, the combination of microscope objectives and cameras is a widely applied optical method, where both a high numerical aperture and a high amplification factor of the objectives are essential for obtaining detailed surface images. However, only the 2D surface information could be recorded by such traditional combination. For obtaining the 3D information of the imaged surface, a type of interferometer should be brought in to include the surface morphology information into interferograms [10], and holography [11] which has been well developed should be used consequently to retrieve it from the interferograms. For re-detecting the surface with other methods to verify the resolution of the retrieved one, the gratings may be good samples under detection due to their high periodicity in height

*corresponding author; e-mail: chengchuanfu@sdnu.edu.cn distribution. Meanwhile, the detection of the morphology of gratings is also of great interest, since the grating has always been one of the most commonly used optical elements and its detailed surface morphology profoundly influences its applications [12].

In this paper, we build an interferometer of MachZehnder type combining a microscope objective of both high numerical aperture and high magnification factor, and detect the surface morphologies of two ruled transmission gratings. The retrieved morphologies of the grating surfaces are compared with the one obtained by an atom force microscope. The results coincide with each other well with nanometer-scaled errors, verifying the feasibility of the non-destructive optical method.

\section{Experiments}

The schematic diagram of the experimental system is shown in Fig. 1. The input beam from a He-Ne laser with wavelength $\lambda=632.8 \mathrm{~nm}$ is split into the reference beam and the object beam with approximately equal power by the beam splitter BS1. The sample of a ruled transmission grating is illuminated perpendicularly by the object

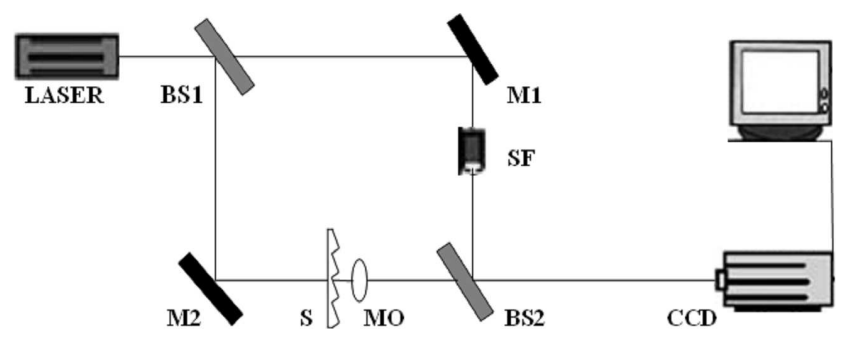

Fig. 1. Experimental setup. BS - beam splitter, $\mathrm{SF}$ - spatial filter, $\mathrm{MO}$ - microscope objective, $\mathrm{S}$ gratings. 
wave in the normal direction, and its surface is magnified and imaged by a microscope objective (N.A. $=0.9$, M. $=100 \times)$. The sample is mounted on a 3D transmission stage for flexible adjustment of the distance between the sample and the microscope objective. With the objective as the imaging lens, a largely magnified real image of the sample with satisfying quality is obtained on the imaging plane. The distance between the sample (lying in the $x O y$ plane) and the objective, to which the magnification is very sensitive, is a little bit smaller than $1 \mathrm{~mm}$, i.e., the work distance of the objective. The magnified image is received by a CCD (Cascade $1 \mathrm{~K}$, pixel size $8 \mu \mathrm{m} \times 8 \mu \mathrm{m}, 1004 \times 1002$ pixels $)$. The reference beam is expended and filtered by a spatial filter SF, which is mounted on a translation stage for precise adjustment of its distance to the receiving plane of the CCD. This adjustment is to eliminate the spherical wave effect in the object wave induced by the large magnification of the objective imaging in which a small area of micrometer scale on the sample surface is extended to a large image covering the CCD. The expanded reference wave is reflected by beam splitter BS2, and it interferes with the extended image of the object. Obviously, the setup constitutes a Mach-Zehnder interferometer. With the spherical wave effect of the object wave eliminated, interferogram with straight interference fringes with equal distances is obtained and recorded by the $\mathrm{CCD}$ on the image plane ( $x O y$ plane). Changing the angle between the object beam and the reference beam by adjusting $\mathrm{BS} 2$, we have vertical interference fringes with appropriate density to distinguish the grating engravings lying in horizontal direction.
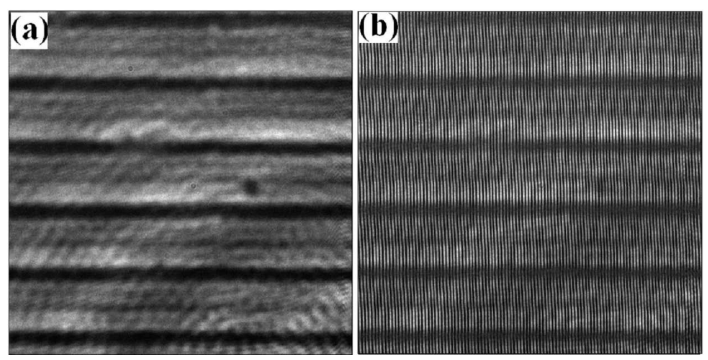

Fig. 2. Images of the grating (a) without and (b) with the reference beam.

In our practical performance, two different ruled transmission gratings of 300 line $/ \mathrm{mm}$ and 600 line $/ \mathrm{mm}$ are used as the samples. By moving the sample for $15 \mu \mathrm{m}$ in vertical direction with respect to the object beam, the pattern in CCD plane move about 820 pixels, equivalent to $6560 \mu \mathrm{m}$. Thus, we deduce that the area of the gratings imaged on the CCD is about $18.3 \mu \mathrm{m} \times 18.3 \mu \mathrm{m}$. Figure $2 \mathrm{a}$ and $\mathrm{b}$ shows the images of the 300 line grating surface without and with the reference wave, respectively. The thick ring-like fringes in Fig. $2 \mathrm{a}$ are induced by the inherent diffraction of the objective to the illuminating laser beam, and its influence may be proved ignorable in our experiment.

\section{Theory and experimental results}

For a transmissive grating with height distribution $h\left(x_{\mathrm{i}}, y_{\mathrm{i}}\right)$ and refractive index $n$ illuminated by a planar light, the phase variation of the light wave immediately after the object is $\Delta \varphi=2 \pi(n-1) h\left(x_{\mathrm{i}}, y_{\mathrm{i}}\right) / \lambda$. As the spherical effect in the object wave induced by the objective has been eliminated by the spherical reference wave and the system is point-to-point imaging, the phase of the object wave at the image plane is $\Delta \varphi=2 \pi(n-1) h\left(X_{\mathrm{i}}, Y_{\mathrm{i}}\right) / \lambda$. The factor $h\left(X_{\mathrm{i}}, Y_{\mathrm{i}}\right)$ can be viewed as $h\left(x_{\mathrm{i}}, y_{\mathrm{i}}\right)$, only with the coordinates extended. Assuming the amplitude transmittance as 1 and normalizing the amplitude of the light to be unity, we express the amplitudes of the object wave and reference light at the image plane respectively as

$$
\begin{aligned}
& U_{1} \propto \exp \left(\mathrm{i} 2 \pi(n-1) h\left(X_{\mathrm{i}}, Y_{\mathrm{i}}\right) \lambda z\right)= \\
& U_{1 R}(X, Y)+\mathrm{i} U_{1 \mathrm{i}}(X, Y), \\
& U_{2} \propto \exp \left(\mathrm{i} 2 \pi\left(X x_{\mathrm{i}}+Y y_{\mathrm{i}}\right) \lambda z\right)= \\
& \quad \exp \left(\mathrm{i} 2 \pi\left(f_{x 0} X+f_{y 0} Y\right)\right),
\end{aligned}
$$

where $U_{1 R}(X, Y)$ and $U_{1 \mathrm{i}}(X, Y)$ are the real and the imaginary parts of the object wave, respectively. Then the intensity at the image plane is expressed as

$$
I=\left|U_{1}+U_{2}\right|^{2}=\left|U_{1}\right|^{2}+\left|U_{2}\right|^{2}+U_{2} U_{1}^{*}+U_{1} U_{2}^{*},
$$

where $*$ denotes complex conjugate. Then the expression of function (3) after Fourier transform is

$$
\begin{aligned}
& I_{f}\left(f_{x}, f_{y}\right)=B_{f}\left(f_{x}, f_{y}\right) \\
& \quad+U_{f}^{*}\left(f_{x}, f_{y}\right) \otimes \delta\left(f_{x}-f_{x 0}, f_{y}-f_{y 0}\right) \\
& \quad+U_{f}\left(f_{x}, f_{y}\right) \otimes \delta\left(f_{x}+f_{x 0}, f_{y}+f_{y 0}\right)=B_{f}\left(f_{x}, f_{y}\right) \\
& \quad+U_{f}^{*}\left(f_{x}-f_{x 0}, f_{y}-f_{y 0}\right)+U_{f}\left(f_{x}+f_{x 0}, f_{y}+f_{y 0}\right),
\end{aligned}
$$

where $\otimes$ denotes the calculation of convolution. $B_{f}\left(f_{x}, f_{y}\right)$ is the zero-order spatial spectrum, which is the Fourier transform of the first and the second terms in the right-hand side of Eq. (3) and corresponds to the central spot in the spatial frequency domain Fig. 3a. The spatial spectra $U_{f}^{*}\left(f_{x}-f_{x 0}, f_{y}-f_{y 0}\right)$ and $U_{f}\left(f_{x}+f_{x 0}, f_{y}+f_{y 0}\right)$ are the Fourier transform of the third and the fourth terms in the right-hand side of Eq. (3), corresponding to the right
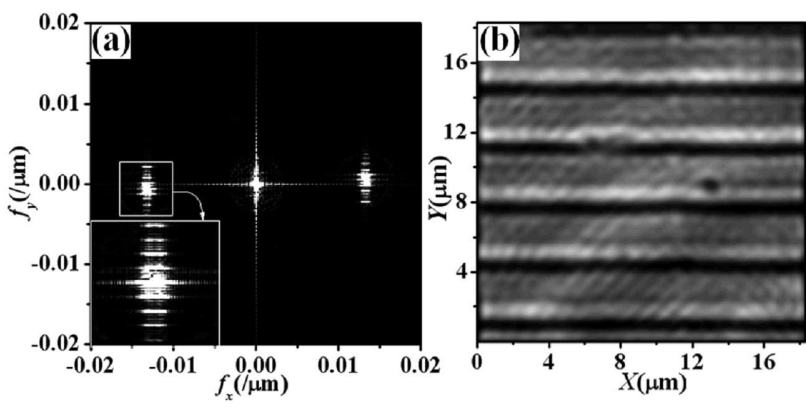

Fig. 3. (a) Spatial spectrum of Fig. 2b. (b) Grating image extracted from Fig. $2 \mathrm{~b}$. 
and the left spots in Fig. 3a, respectively. It is noted that the two spots approximately consist of several separate spectra corresponding to different diffraction orders of the grating, lying in the $f_{x}$ direction. We could obtain the amplitude of the objective wave by certain processes on either of the two spots, and in our experiment the left spot is taken. In order to include the details of the grating surface and simultaneously eliminate the influence of optical noises, the spot is taken in an appropriate spectral range (marked within the square frame and shown for details at the corner of Fig. 3a). The selected spot is translated along $f_{x}$ direction by $f_{x 0}$ and along $f_{y}$ direction by $f_{y 0}$ to eliminate the influence of the reference wave, and is now expressed to be $U_{f}\left(f_{x}, f_{y}\right)$. Then the calculation of inverse Fourier transform is performed on it, and the final result is right $U(X, Y)$, the amplitude of the object wave at the image plane.

During the above reconstruction processes, the real part $U_{1 R}(X, Y)$ and the imaginary part $U_{1 \mathrm{i}}(X, Y)$ of the amplitude are extracted separately. Then the intensity distribution of the object wave is written as $I(x, y)=$ $U_{1 R}^{2}(X, Y)+U_{1 \mathrm{i}}^{2}(X, Y)$ and the image of such extracted intensity pattern is depicted in Fig. 3b, which is similar with the image recorded directly by CCD (Fig. 2a). Noticeably, the definition of the extracted image is much higher than the recorded one, as the adverse influence of optical noises with high spatial frequency has been eliminated during the processes. The phase distribution of the wave at the image plane can be obtained by $\varphi(X, Y)=\arctan \left(U_{1 \mathrm{i}}(X, Y) / U_{1 R}(X, Y)\right)$. As the phase maps may contain $2 \pi$ discontinuities, phase unwrapping processes should be adopted in digital holography. Among the well-developed phase unwrapping method, the simple algorithm in [13] is used in our paper to realize such process, and even the global discontinuities contained by the phase maps can also be unwrapped correctly herein, which may be the major advantage of this algorithm. Then, the accurate phase distribution of the waves immediately behind the 300 line grating surface is obtained $\varphi(x, y)$, by merely downscaling the coordinates $(X, Y)$ to $(x, y)$.

We show the phase distribution $\varphi(x, y)$ in Fig. 4a in an area of $18.3 \mu \mathrm{m} \times 18.3 \mu \mathrm{m}$. Herein, the phase contours are horizontal and fluctuate periodically in vertical direction. Consequently, the height distribution of the grating surface is obtained by

$$
h\left(x_{\mathrm{i}}, y_{\mathrm{i}}\right)=\lambda \varphi\left(x_{\mathrm{i}}, y_{\mathrm{i}}\right) / 2 \pi(n-1),
$$

which is proportional to the phase values. Obviously, the height distribution of the grating surface fluctuates in the same manner as in Fig. 3a. For clear seen, the height distribution along the vertical direction is depicted in Fig. 4b. We see that the average height step from the lowest points to the highest points is $h=298 \mathrm{~nm}$ and the grating constant is $a=3.341 \mu \mathrm{m}$. To see the height fluctuation more clearly, a stretched illustration is depicted at the left corner of Fig. $4 \mathrm{~b}$, and it is found that the grating surface is just like some mountains with steep cliffs on one side and gentle slopes on the other side.
In order to examine the detection accuracy, we then detect the 300 line grating surface by an atomic force machine and show the result in Fig. 5a. From its height distribution in vertical direction shown in Fig. 5b, we find that the average height step of the 300 line grating surface is about $h=293 \mathrm{~nm}$. That is, the relative error of the height steps between the two methods is only about $1.68 \%$. Moreover, the fluctuation styles obtained in the two methods coincide with each other well, as can be seen by comparing Fig. 4b with Fig. 5b. This indicates that the results we obtained in the non-destructive way have satisfactory precision.
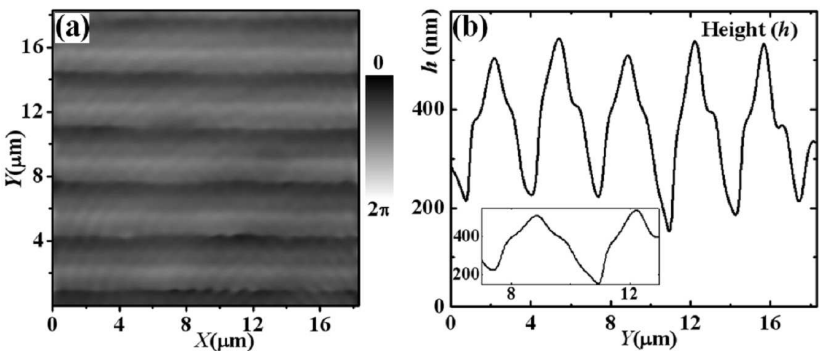

Fig. 4. (a) Phase distribution in the wave right behind the 300 line $/ \mathrm{mm}$ grating. (b) Height distribution of the grating.
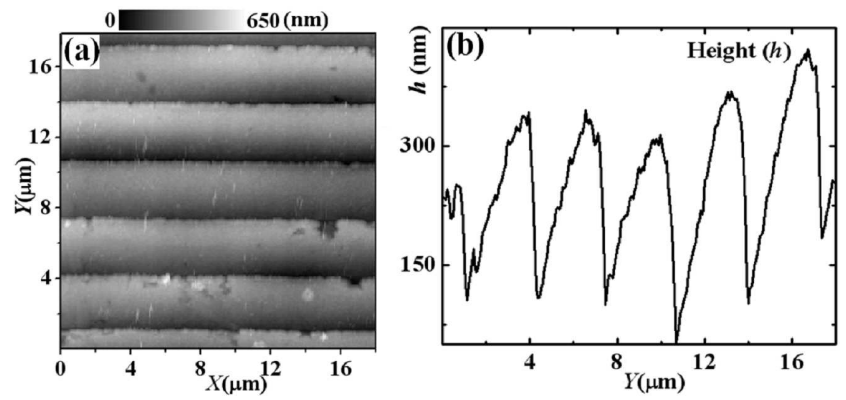

Fig. 5. (a) AFM image of the 300 line $/ \mathrm{mm}$ grating.

(b) Height distribution versus vertical direction.
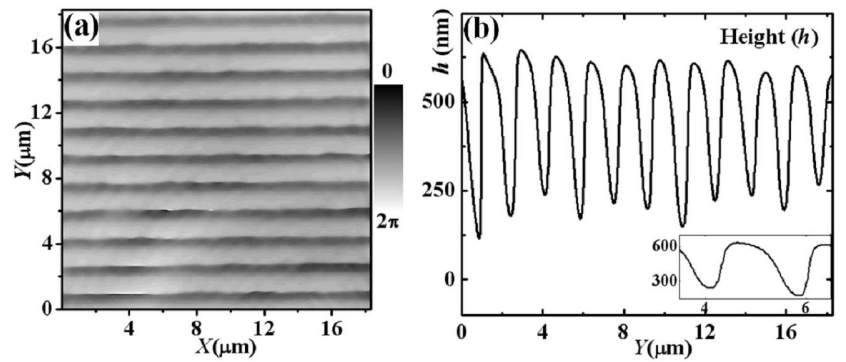

Fig. 6. (a) Phase distribution in the wave right behind the 600 line/mm grating. (b) Height distribution of the grating.

The morphology of a 600 line/mm ruled transmission grating is also detected. From the phase distribution shown in Fig. 6a, we obtain its height distribution versus the vertical coordinate and show it in Fig. 6b. We note 
that the average height step from the highest points to the lowest points is about $421 \mathrm{~nm}$ and the grating constant is $1.673 \mu \mathrm{m}$, about half of that of the 300 line grating. Noticeably, the curve in Fig. $6 \mathrm{~b}$ is not so acute as the curve in Fig. 4b. This can be explained as follows. For a grating with grating constant $a$, the maximum diffraction order that can be captured by the objective is calculated by $k=a \sin \theta / \lambda$, where $\theta=72^{\circ}$ is the acceptance angle of the objective we use. Accordingly, nine diffraction orders of the 300 line grating may be captured by the objective; but for the 600 line grating, the acceptable diffraction orders may be lower, e.g., the $( \pm 2, \pm 1,0)$ th orders, while the higher orders with diffraction angles larger than $72^{\circ}$ cannot be captured. That is, the wave components of higher frequency are missed, and this leads to the lower resolution in Fig. $6 \mathrm{~b}$.

\section{Discussions and conclusions}

The surface morphology of an opaque object may also be obtained by using the microscope objective. However, the light illuminating the object should be reflected back therein, rather than passing through, before it comes to the image plane. From another perspective, when the thickness distribution of a sample is known, the refractive index distribution of the sample may be easily obtained as $n=2 \pi h / \lambda \varphi+1$. This may be a useful method to detect the components of living cells without doing damages to them, for the refractive index of a cell is interrelated with its components.

In conclusion, the Mach-Zehnder interferometer is combined with an optical microscope objective of high numerical number, and the surfaces of two ruled transmission gratings are detected. From the interferogram constructed by the spherical reference beam and the objective wave, we retrieved both the intensity distributions of the object waves in the image plane and the phase maps of the object waves right behind the grating surfaces. The morphologies of the grating surfaces in small areas of $\mu \mathrm{m} \times \mu \mathrm{m}$ scale are calculated accordingly. In summary, we realize the 3D detection with a common optical microscope objective that was used to magnify 2D images traditionally. This cheap, convenient and non-destructive optical tool has achieved the precision scaled to nm.

\section{Acknowledgments}

Supported by the National Natural Science Foundation of China (Grant No. 10974122), the Shandong Distinguished Middle-Aged and Young Scientist Encourage and Reward Foundation, China (Grant No. BS2009SF020).

\section{References}

[1] M. Python, E. Vallat-Sauvain, J. Bailat, D. Dominé, L. Fesquet, A. Shah, Ch. Ballif, J. Non-Cryst. Solids 354, 2258 (2008).

[2] H. Ohta, K. Nomura, H. Hiramatsu, K. Ueda, T. Kamiya, M. Hirano, H. Hosono, Solid-State Electron. 47, 2261 (2003).

[3] Y. Tsuboi, R. Shimizu, T. Shoji, N. Kitamura, J. Am. Chem. Soc. 131, 12623 (2009).

[4] H. Liu, B. Wang, E.S.P. Leong, P. Yang, Y. Zong, G.Y. Si, J.H. Teng, S.A. Maier, ACS Nano 4, 3139 (2010).

[5] S. Sarkar, S. Patra, N. Gayathri, S. Banerjee, Appl. Phys. Lett. 96, 063112 (2010).

[7] F.J. Giessibl, Rev. Mod. Phys. 75, 949 (2003).

[8] J.M. Burch, Nature 171, 889 (1953).

[9] I.K. Robinson, D.J. Tweet, Rep. Prog. Phys. 55, 599 (1992).

[10] M.K. Kim, L.F. Yu, C.M. Lo, C.J. Mann, em Opt. Expr. 13, 8693 (2005).

[11] E. Cuche, F. Bevilacqua, C. Depeursinge, Opt. Lett. 24, 291 (1999).

[12] T. Weitkamp, A. Diaz, C. David, F. Pfeiffer, M. Stampanoni, P. Cloetens, E. Ziegler, Opt. Expr. 13, 6296 (2005).

[13] J.M. Huntley, H. Saldner, Appl. Opt. 32, 3047 (1993). 